\title{
Estimación de riesgo de enfermedad coronaria mediante la función de Framingham adaptada para la población chilena
}

\author{
G loria Icaza ${ }^{1, a b}$, Loreto N úñez 2,ac, Jaume Marrugat ${ }^{3}$, \\ Verónica Mujica 4 ,a, M. Cristina Escobar ${ }^{5}$, \\ Ana Luisa Jiménez ${ }^{6, a d}$, Paulina Péreze ${ }^{\mathrm{e}}$ Iván Palomo ${ }^{4, a f}$. \\ Estimation of coronary heart disease \\ risk in Chilean subjects based on \\ adapted Framingham equations
}

Background: Cardiovascular disease is the leading cause of morbidity and mortality in Chile and worldwide. Framingham functions were developed to calculate overall coronary heart disease risk. However these functions overestimate the risk in some countries like Chile. Aim: To develop Chilean risk tables to assess the overall 10-year risk of coronary heart disease. Material and methods: The Framingham function was adapted for a population aged 35 to 74 years, based on an estimate of Chilean incidence of coronary heart disease and the prevalence of coronary heart disease risk factors such as age, sex, total cholesterol, high-density lipoprotein cholesterol, blood pressure, diabetes and smoking. Results: The 10-year incidence of coronary heart disease in Chile ( $2.7 \%$ in men, $1.0 \%$ in women) was lower than the incidence in the United States $(10.0 \% / 3.8 \%)$ and Spain $(4.9 \% / 2.2 \%)$, but higher than China $(1.1 \% / 0.4 \%)$. Framingham tables have more than $50 \%$ of cells in the risk category of $10 \%$ or greater. In contrast, Chilean tables have less than $10 \%$ of cells in the same risk category. Conclusions: Adapted tables use local information to calculate overall coronary heart disease risk. A validation study should be conducted to assess their predictive power (Rev Méd Chile 2009; 137: 1273-82).

(Key w ords: Coronary artery disease; Epidemiology; Risk factors)

Recibido el 29 de enero, 2009. Aprobado el 27 de agosto, 2009.

Trabajo financiado por FONIS, proyecto \# SA06I20065

${ }^{1}$ Instituto de Matemática y Física, Universidad de Talca. Talca, Chile. ${ }^{2}$ Departamento de Salud Pública, Facultad de Ciencias de la Salud, Universidad de Talca. Talca, Chile. ${ }^{3}$ Unitat de Lípids i Epidemiologia Cardiovascular, Programa de Recerca en Processos Inflamatoris i Cardiovasculars, Institut Municipal d'Investigació Medica, Barcelona, España. ${ }^{4}$ Departamento de Bioquímica Clínica e Inmunohematología, Facultad de Ciencias de la Salud, Universidad de Talca. Talca, Chile. ${ }^{5}$ Departamento de Enfermedades no Transmisibles, División de Prevención y Control de Enfermedades, Ministerio de Salud, Chile. ${ }^{6}$ Centro de Salud Astaburuaga, Talca, Chile.

a Programa de Investigación de Factores de Riesgo de Enfermedades Cardiovasculares de la Universidad de Talca

${ }^{b}$ Estadística, PhD

${ }^{\mathrm{c}}$ Cirujano Dentista, MPH

${ }^{\mathrm{d}}$ Enfermera, MPH

eEstadística

f Tecnólogo Médico, PhD

Correspondencia a: Gloria Icaza. Instituto de Matemáticas y Física, Universidad de Talca. Casilla 721, Talca, Chile. Teléfono: 5671200 338. Fax: 5671200 392. E mail: gicaza@utalca.cl 
$\mathrm{L}^{2}$ a Organización Mundial de la Salud (OMS) estimó que en el año 1996 murieron en el mundo unos 15 millones de personas por alguna enfermedad cardiovascular (ECV), lo que representa $29 \%$ de la mortalidad total, siendo la cardiopatía isquémica la responsable de la muerte de 7 millones de personas ${ }^{1}$. En Chile las ECV representan un tercio de la mortalidad total y son además una de las principales causas de morbilidad; en 2005 se produjeron 110.138 egresos por enfermedades del sistema circulatorio ${ }^{2}$, por lo que el control y compensación de los usuarios con patología cardiovascular constituye una de las principales metas sanitarias para el Ministerio de Salud ${ }^{3}$. Se estima que más de 50\% de los problemas que originan las ECV podrían evitarse si se logra reducir la incidencia a través de la prevención de sus factores de riesgo ${ }^{1}$. El estudio de Framingham ha contribuido a la identificación de los factores de riesgo de enfermedades isquémicas del corazón y otras $\mathrm{ECV}^{4,5}$. Como producto del análisis del estudio de Framingham, se han publicado modelos que permiten, a los clínicos, la estimación de la probabilidad de que un individuo desarrolle un acontecimiento coronario a 10 años $^{6}$. Este cálculo se realiza a partir de los factores de riesgo coronario mayores como: diabetes, hipertensión arterial (HTA), dislipidemia, tabaquismo, sexo masculino y $\operatorname{edad}^{7,8}$.

Desde el año 2002, Chile cuenta con el Programa de Salud Cardiovascular del Ministerio de Salud, mediante el cual se cambió el enfoque terapéutico, desde uno dirigido a tratar factores de riesgos aislados (HTA, diabetes, tabaquismo o dislipidemia), a otro que considera el riesgo global de ECV del individuo. El Programa de Salud Cardiovascular propone dos alternativas para evaluar el riesgo de ECV: una cuantitativa, utilizando los puntajes de riesgo del estudio de Framingham ${ }^{7}$ para predecir riesgo coronario a 10 años o una cualitativa que considera el número de factores de riesgo cardiovascular mayores modificables: HTA, diabetes, tabaquismo y dislipidemia?. Ambos instrumentos de evaluación permiten clasificar a los individuos en cuatro categorías de riesgo (bajo, moderado, alto o máximo), lo que determina, a su vez, las metas e intervenciones terapéuticas ${ }^{10}$.

Para el cálculo del riesgo coronario se han desarrollado diferentes algoritmos matemáticos como el de Framingham ${ }^{7,8}$ y tablas $^{4,11-13}$ que estiman el exceso de riesgo del individuo con respecto al promedio de la población a la que pertenece y constituyen una herramienta para establecer prioridades en prevención primaria ${ }^{14}$.

Originalmente estos algoritmos se aplicaron en guías clínicas de prevención de ECV por parte de sociedades científicas de Estados Unidos de Norteamérica y Europa. Sin embargo, se ha constatado que la función de Framingham sobreestima el riesgo coronario en ciertas poblaciones de Estados Unidos de Norteamérica y en países con una menor incidencia de enfermedad coronaria, tales como: Alemania, Italia, España, Dinamarca y Chi$\mathrm{na}^{5,15-20}$. Chile no dispone de estimaciones poblacionales de incidencia de enfermedad coronaria. Sin embargo, por ser un país que comparativamente tiene una baja mortalidad por infarto agudo al miocardio (IAM) ${ }^{21}$, se podría deducir que la función de Framingham también sobreestimaría el riesgo coronario a 10 años de la población chilena.

D'Agostino et $\mathrm{al}^{15}$ propusieron que, de no existir estudios poblacionales de cohorte para generar estimaciones de riesgo coronario local, se podía adaptar la función de Framingham, considerando la prevalencia local de factores de riesgo de ECV y la incidencia de acontecimientos coronarios. Este método fue aplicado, y luego validado, en España ${ }^{4,22}$ y en China $^{5}$, ambos países con baja incidencia de eventos coronarios.

El objetivo general de este estudio fue adaptar la función de Framingham para la población chilena de 35 a 74 años y generar tablas nacionales de estimación de riesgo coronario global a 10 años.

\section{MATERIAL Y MÉTOdO}

Se adaptó la función de riesgo coronario de Framingham de Wilson et $\mathrm{al}^{8}$, siguiendo la propuesta metodológica de D'Agostino et $\mathrm{al}^{15}$ y Marrugat et $\mathrm{al}^{4}$.

Los eventos coronarios que considera la función de Framingham son IAM mortal (hospitalizado o no); IAM no mortal (con síntomas y sin síntomas) y angina ${ }^{8}$. Entre estos eventos se distinguen el IAM mortal y el IAM no mortal con síntomas, llamados eventos mayores (hard endpoints), los cuales son susceptibles de ser medidos a escala poblacional.

Los factores de riesgo incluidos en la función de Wilson ${ }^{8}$ son: edad, sexo, colesterol total, colesterol unido a lipoproteínas de alta densidad (cHDL), presión arterial, diabetes y tabaquismo (Tabla 1). 


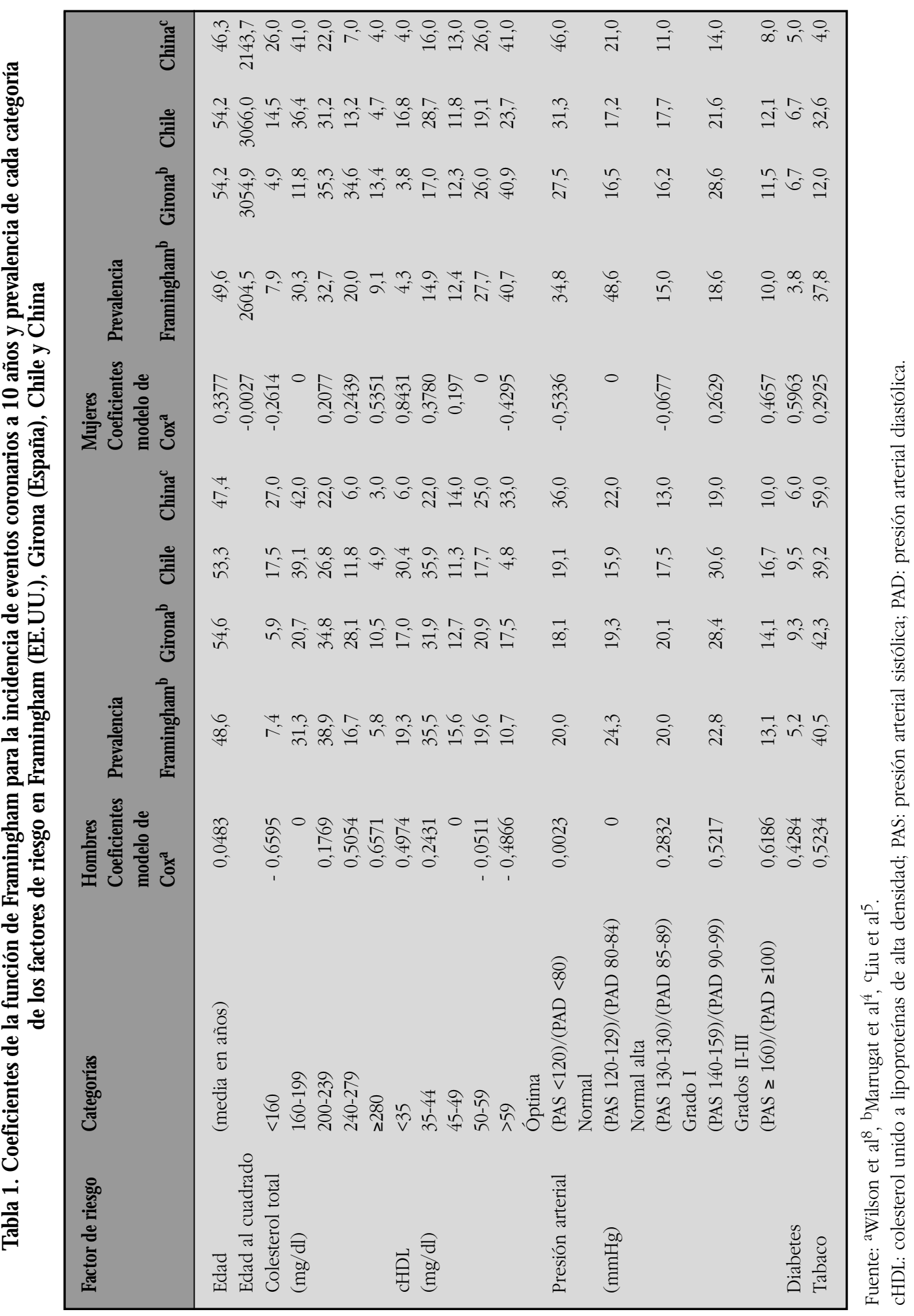


El modelo utilizado para generar la función de Framingham $^{8}$ se conoce como modelo de riesgos proporcionales de Cox, a partir del cual se puede estimar la probabilidad de tener un acontecimiento coronario en 10 años. Esta probabilidad depende de dos variables, la incidencia de eventos coronarios y la prevalencia de factores de riesgo de la población. Asumiendo que el riesgo relativo para cada factor de riesgo es el mismo en distintas poblaciones $^{22}$, se sustituye, en la función original de Wilson ${ }^{8}$, la incidencia estimada de eventos coronarios y el riesgo medio poblacional de Chile, basado en la prevalencia de los factores de riesgo considerados (Tabla 1).

La prevalencia de factores de riesgo para el grupo de 35 a 74 años de edad, se calculó de la Encuesta Nacional de Salud $2003^{23}$, para lo cual se tuvo acceso a la base de datos a través de un convenio con el Departamento de Epidemiología del Ministerio de Salud. La Encuesta Nacional de Salud 2003 es una muestra representativa de la población general mayor de 17 años de todo el país.

Para la estimación de la incidencia de IAM mortal y no mortal con síntomas (eventos mayores), se utilizaron las Estadísticas de Mortalidad y Egresos Hospitalarios del Ministerio de Salud entre los años 2001 y 2005², códigos I21-I22 de la décima revisión de la Clasificación Estadística Internacional de Enfermedades (CIE10) ${ }^{24}$. Las estadísticas oficiales chilenas, especialmente las Estadísticas de Mortalidad, han sido consideradas históricamente de buena calidad, no obstante persisten problemas con causas mal codificadas 25 . Por lo anterior se corrigieron los datos de mortalidad adicionando dos variables: (a) el porcentaje de causas mal definidas totales, códigos R00-R99 de la CIE10 y (b) frecuencia de ECV sin significado diagnóstico, códigos I47.2, I49.0, I46, I50, I51.4, I51.5, I51.6, I51.9, I70.9 de la CIE10 ${ }^{26}$. Por otra parte, el reporte de los egresos hospitalarios está considerado en el Código Sanitario ${ }^{27}$, se estima que tiene una cobertura de 95\% de los egresos, por lo cual se realizó una corrección, asumiendo de manera conservadora que este 5\% podría corresponder a IAM. Con estos datos se estimó la tasa de incidencia acumulada 28 de eventos mayores, como la sumatoria de egresos hospitalarios vivos por IAM corregidos, más la mortalidad por IAM corregida, todo esto dividido por la población estimada del año correspondiente según proyecciones de población del censo $2002^{2}$. Para la estimación de incidencia de IAM silente y angina, se asumió una proporción similar a la de Framingham, por lo cual se corrigió la incidencia de eventos mayores multiplicándola por 1,40 para hombres y 1,91 para mujeres ${ }^{4}$.

Una vez obtenida la incidencia de eventos coronarios y la prevalencia de factores de riesgo locales, se adaptó la función de Framingham, desarrollándose tablas y un programa computacional de cálculo de riesgo coronario a 10 años en línea. Las tablas separan diabéticos y no diabéticos, hombres y mujeres, fumadores y no fumadores. Cada casilla contiene el riesgo calculado aproximando al entero más alto. Se utilizaron puntos de corte para categorías de riesgo usados en otras tablas ${ }^{4,29}$, quedando cuatro categorías de riesgo: bajo $\leq 5 \%$, moderado 6\%-9\%, alto 10\%-19\% y muy alto $\geq 20 \%$. Estas tablas están hechas para un valor de cHDL entre 35 y $59 \mathrm{mg} / \mathrm{dl}$. El riesgo puede ser corregido para valores de cHDL fuera de este rango.

Se comparó la distribución de frecuencias de las casillas de riesgo de la tabla original de Wilson $^{8}$ y las adaptaciones en España ${ }^{4}$, China ${ }^{5}$ y Chile mediante el diagrama de cajas (boxplot), en que la línea horizontal en la caja representa la mediana de los datos y el alto de las cajas representa el rango entre los percentiles 75 y 25 .

El cálculo de riesgo coronario se realizó con el programa SAS versión 9.1.3.

\section{Resultados}

La prevalencia de factores de riesgo en población chilena de 35 a 74 años es distinta en comparación con la encontrada en las poblaciones de Estados Unidos de Norteamérica (Framingham), España (Girona) y China, tanto en hombres como mujeres (Tabla 1). La incidencia de eventos coronarios a 10 años también es diferente, Chile presenta menor incidencia que la población de Framingham y Girona, es similar a hombres hispanos de Estados Unidos de Norteamérica y es superior a la de China (Figura 1). En todas las poblaciones comparadas, la incidencia de eventos coronarios a 10 años es mayor en hombres que en mujeres. 


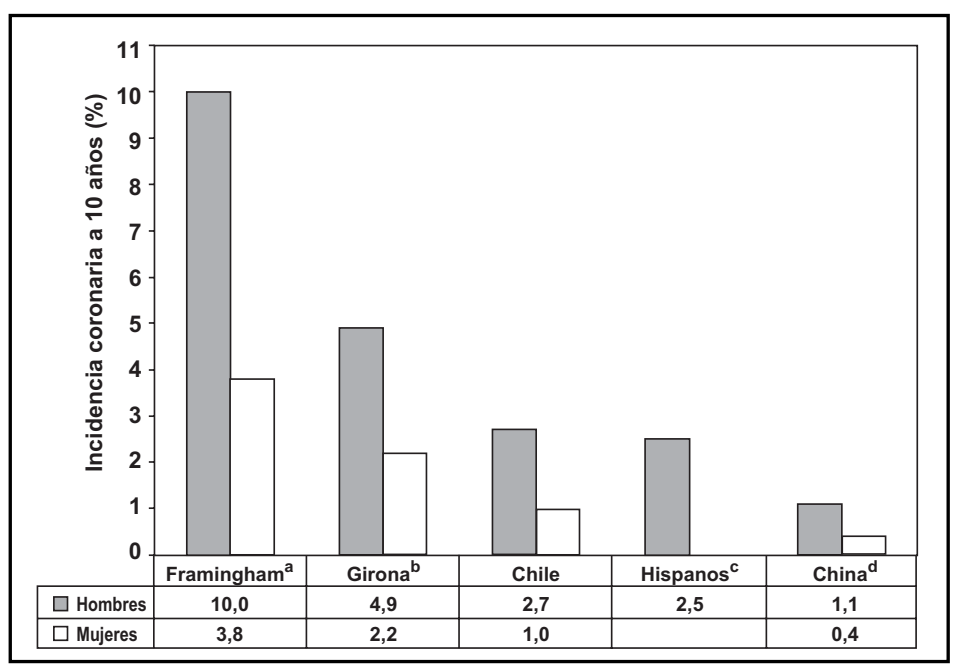

Figura 1. Comparación de incidencia de eventos coronarios a 10 años en distintas poblaciones de Estados Unidos de Norteamérica, España, Chile y China.

En las tablas de estimación de riesgo coronario a 10 años para población de 35 a 74 años hay paneles diferentes para cada sexo y para personas diabéticas y no diabéticas (Figuras 2 y 3). Se aprecia el mayor riesgo coronario para diabéticos y fumadores y el menor riesgo para mujeres no diabéticas.

Al comparar los valores de riesgo de cada casilla de las tablas de riesgo coronario a 10 años de las poblaciones estudiadas, Framingham presenta más de $50 \%$ de casillas en categorías de riesgo mayor o igual a $10 \%$, seguido por Girona donde alrededor de $25 \%$ de las casillas se encuentran en dicha categoría, Chile donde menos de $10 \%$ de las casillas se encuentran en esa categoría de riesgo y finalmente, la tabla correspondiente a China presenta menos de 5\% de casillas con riesgo mayor o igual a $10 \%$ (Figura 4).

\section{DISCUSIÓN}

La recomendación de la OMS es realizar esfuerzos en los países para prevenir la ECV basados en el riesgo de tener un evento coronario a 10 años, por lo cual contar con un instrumento que estime el riesgo permitirá establecer acciones clínicas en aquellos indi- viduos que tienen mayor probabilidad de verse beneficiados ${ }^{30}$.

La adaptación que se realizó en este estudio es una forma de contar con información local que permita tomar decisiones para la prevención primaria de la ECV. Sin embargo, hay que ser prudentes en el uso de estas tablas, debido a que lo realizado es una extrapolación de una función de cálculo de riesgo en población anglosajona y no se sabe si los riesgos relativos del estudio de Framingham se reproducen en la población chilena. No obstante, en Girona se realizó la validación de las tablas adaptadas con la misma metodología de este estudio, la cual mostró que los riesgos relativos de la función de Framingham se reproducían en la población española ${ }^{19}$. Información similar muestra el estudio de China ${ }^{5}$. Es imperativo que se realice un estudio de validación prospectiva que mida el poder predictivo de estas tablas en la población chilena ${ }^{4,5}$.

Por otro lado, no disponemos de registros poblacionales para el cálculo de incidencia de enfermedad coronaria. En todo caso, la magnitud de la estimación de incidencia era esperable, debido a las características demográficas y de mortalidad por cardiopatía isquémica en Chile, ya que por tratarse de una población más joven que la europea o norteamericana, la mortalidad no estandarizada es menor que en esas poblacio- 


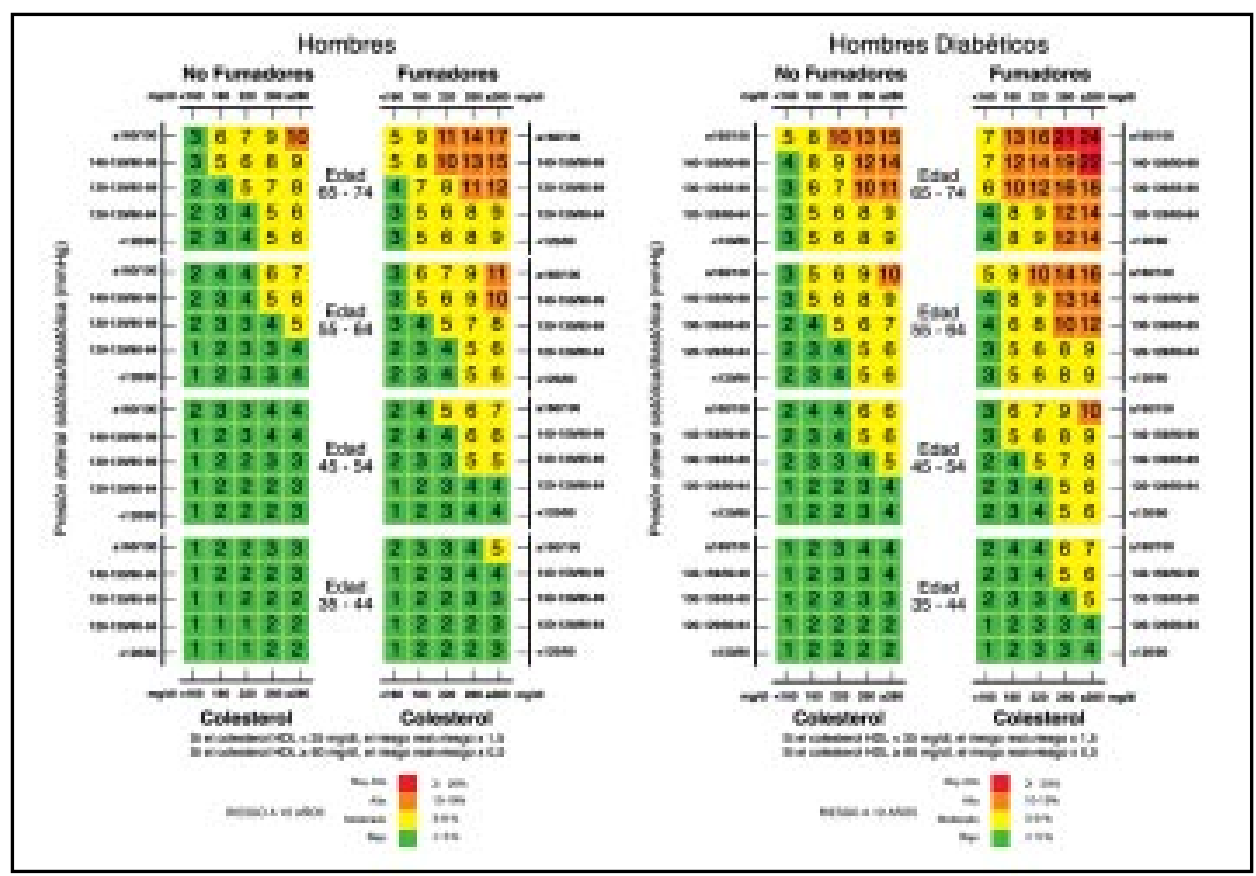

Figura 2. Tabla de estimación de riesgo coronario a 10 años en hombres diabéticos y no diabéticos de 35 a 74 años para población chilena.

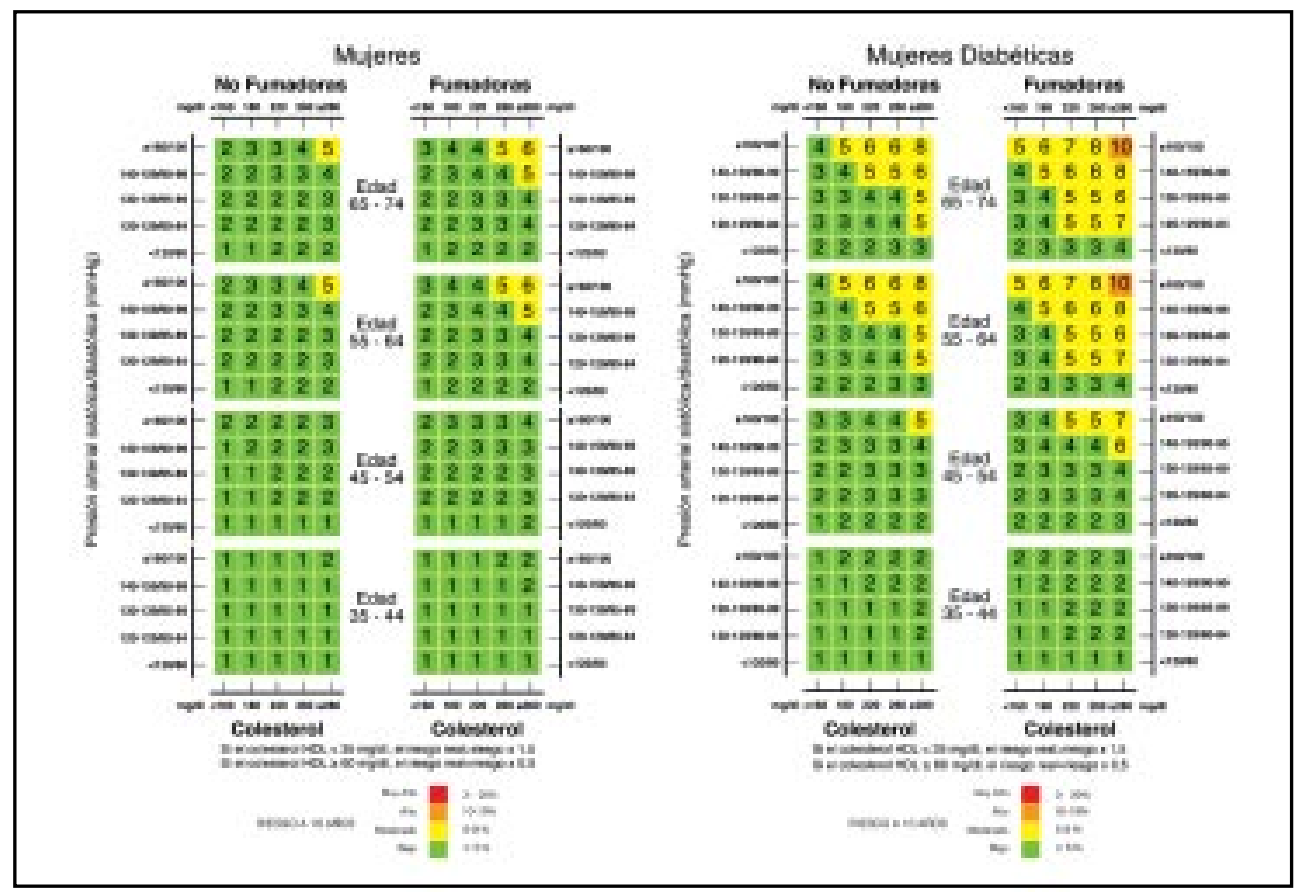

Figura 3. Tabla de estimación de riesgo coronario a 10 años en mujeres diabéticas y no diabéticas de 35 a 74 años para población chilena. 


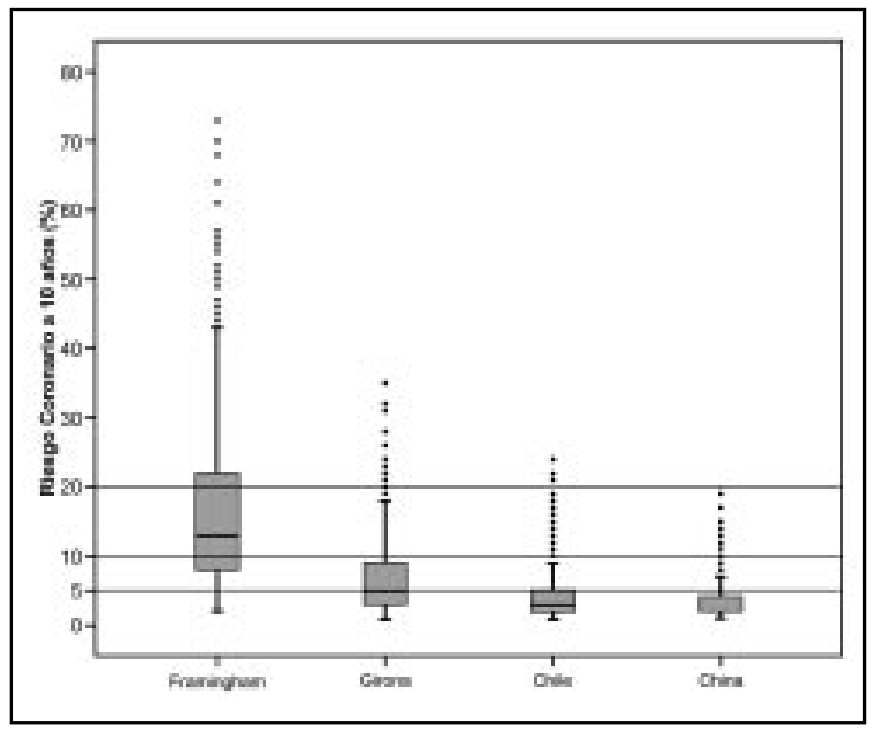

Figura 4. Comparación de puntajes de casillas de riesgo coronario a 10 años en distintas tablas desarrolladas en Framingham (EE.UU.), Girona (España), Chile y China.

nes $^{31}$. En este mismo sentido, la aproximación de incidencia de IAM silente y angina sobre los acontecimientos mayores es conservadora, ya que es poco probable que esta proporción sea mayor en Chile que en Framingham.

Recientemente, los investigadores de Framingham crearon una nueva función para la estimación del riesgo global de ECV que incluye cardiopatía isquémica, accidente vascular cerebral, enfermedad vascular periférica y muerte súbita ${ }^{32}$. En el futuro, deberían incluirse un mayor número de eventos cardiovasculares para la elaboración de tablas predictivas en Chile.

Otro aspecto importante que debe tenerse presente, es que el uso de este tipo de tablas tiene ciertas limitaciones, están diseñadas para prevención primaria, por lo cual no son aplicables en personas que ya han tenido algún evento cardiovascular; en este sentido la inclusión de la diabetes entre los factores de riesgo está aún en discusión, ya que algunos autores sugieren que la diabetes debe considerarse como un equivalente coronario $9,33-41$.

Por otra parte, a pesar de los considerables avances en el tratamiento de las enfermedades cardiovasculares, aún son la principal causa de muerte de los países desarrollados. El uso de instrumentos de evaluación de riesgo coronario permite realizar un tamizaje o cribado de prevención primaria basado en pruebas científicas. Sin embargo algunos individuos de la población que desarrollarán enfermedad coronaria no presentan factores de riesgo clásicos, por lo que se están explorando nuevos biomarcadores como el grosor de la íntima media carotídea, el índice tobillo brazo, la proteína $C$ reactiva ultrasensible, y determinantes genéticos entre otros ${ }^{42}$.

Estas tablas servirán como insumo para guías clínicas de prevención primaria para evaluar riesgo coronario a 10 años con el fin de orientar las prioridades de la prevención primaria de ECV. Es así como en España las tablas adaptadas por el grupo REGICOR ${ }^{4}$, son utilizadas como parte de guías clínicas para prevención primaria de $\mathrm{ECV}^{43,44}$ y son particularmente útiles para la toma de decisiones de inicio de terapia farmacológica de la dislipidemia ${ }^{14}$. Por otro lado, la Comisión de Sanidad y Consumo del Senado de España, ha aprobado una moción que insta el uso de las tablas de Girona para la prevención de ECV en toda España ${ }^{45}$.

Las tablas y un programa de cálculo de riesgo coronario a 10 años en línea, se encuentran disponibles en el sitio web del Programa de Investigación de Factores de Riesgo de Enfermedades Cardiovasculares de la Universidad de Talca 
(http://pifrecv.utalca.cl). Estos productos podrán integrarse al sistema nacional de salud como orientación mientras se desarrolla el proceso de validación. Ello contribuirá a la familiarización con este tipo de instrumentos por parte de los clínicos responsables de la prevención primaria de la ECV.

Se espera que este estudio sirva de estímulo para realizar ecuaciones propias para la población chilena a través de la realización de estudios de cohorte. Hay que notar que en zonas de baja incidencia, como Chile, serán necesarias cohortes muy grandes para acumular, a 10 años, un número suficiente de eventos cardiovasculares, de modo que las estimaciones de los coeficientes de los modelos sean fiables $\mathrm{y}$ precisas $^{46}$. Sin embargo, en poblaciones donde no se cuente con estudios de cohorte, la adaptación de la función de Framingham para desarrollar tablas de

\section{REFERENCIAS}

1. Organización Mundial de la Salud. Informe sobre la salud en el mundo 2002: Reducir los riesgos y promover una vida sana. Ginebra: OMS; 2002. Disponible en: http://www.who.int/whr/2002/es/ index.html [Consultado el 12 mayo 2008].

2. Ministerio de Salud. Departamento de Estadísticas e Información en Salud. Disponible en: http:// deis.minsal.cl [Consultado el 20 mayo 2008].

3. Ministerio de Salud. Los objetivos sanitarios de la década 2000-2010. Gobierno de Chile, 2002. Disponible en: http://epi.minsal.cl/epi/html/sdesalud/OS/ OS2000_2010.htm [Consultado el 25 marzo 2008].

4. Marrugat J, Solanas P, D'Agostino RB, Sullivan L, Ordovas J, Cordón F ET al. Estimación del riesgo coronario en España mediante la ecuación de Framingham calibrada. Rev Esp Cardiol 2003; 56: 253-61.

5. Liu J, Hong Y, D'Agostino RB, Wu Z, Wang W, Sun J ET AL. Predictive value for the Chinese population of the Framingham CHD risk assessment tool compared with the Chinese multi-provincial cohort study. JAMA 2004; 291: 2591-9.

6. Kannel WB, D'Agostino RB, Sullivan L, Wilson PW. Concept and usefulness of cardiovascular risk profiles. Am Heart J 2004; 148: 16-26.

7. Anderson K, Wilson PW, Odell P, Kannel WB. An updated Coronary Risk Profile. A Statement for Health Professionals. Circulation 1991; 83: 35662.

8. Wilson PW, D'Agostino RB, Levy D, Belanger A, Silbershatz H, Kannel WB. Prediction of coronary riesgo coronario puede ser un método efectivo para la prevención primaria de la $\mathrm{ECV}^{4,5}$.

\section{Agradecimientos}

Los autores agradecen a: Dra. Danuta Raj por facilitar las bases de datos de mortalidad y egresos y proporcionar información relevante para el uso de esta información; Joan Vila, estadístico del Institut Municipal d'Investigació Medica (IMIM) de Barcelona, España por su ayuda en la revisión de los cálculos; los académicos y clínicos Dra. Liliana Jadue, Iris Delgado, Dra. Paula Margozzini, Carolina Nazzal, Dr. Gonzalo Díaz, Dr. Patricio Maragaño por sus generosos aportes durante el desarrollo de este proyecto.

heart disease using risk factor categories. Circulation 1998; 97: 1837-47.

9. Expert Panel on Detection, Evaluation, and Treatment of High Blood Cholesterol in Adults. Third report of the National Cholesterol Education Program (NCEP) (Adult Treatment Panel III) final report. Circulation 2002; 106: 3143-421.

10. Ministerio de Salud. Programa de Salud Cardiovascular: Reorientación de los programas de hipertensión y diabetes. Ministerio de Salud, 2002. Disponible en: http://www.redsalud.gov.cl/archivos/saludcardiovascular/Reorientacion_de_los_Programas_de_Hipertension_y_Diabetes.pdf [Consultado el 14 mayo 2007].

11. JACKSON R. Updated New Zealand cardiovascular disease risk benefit prediction guide. BMJ 2000; 320: 709-10.

12. Wood D, De Backer G, Faergeman O, Graham I, Mancia G, Pyörala K. Task Force Report. Prevention of coronary heart disease in clinical practice: Recommendations of the second joint task force of the joint European Societies on coronary prevention. Eur Heart J 1998; 19: 1434-503.

13. Conroy RM, Pyörälä K, Fitzgerald AP, Sans S, Menotti A, De Backer G et al. Estimation of tenyear risk of fatal cardiovascular disease in Europe: the SCORE project. Eur Heart J 2003; 24: 987-1003.

14. Grau M, Marrugat J. Funciones de riesgo en la prevención primaria de las enfermedades cardiovasculares. Rev Esp Cardiol 2008; 61: 404-16.

15. D'Agostino RB, Grundy S, Sullivan LM, Wilson PW. Validation of the Framingham Coronary Heart Disea- 
se Prediction Scores: Results of a Multiple Ethnic Groups Investigation. JAMA 2001; 286: 180-7.

16. Assmann G, Cullen P, Schulte H. Simple scoring scheme for calculating the risk of acute coronary events based on the 10-year follow-up of the prospective cardiovascular Münster (PROCAM) study. Circulation 2002; 105: 310-5.

17. Thomsen T, Mcgee D, Davidsen M, Jørgensen T. A cross-validation of risk-scores for coronary heart disease mortality based on data from the Glostrup population studies and Framingham heart study. Int J Epidemiol 2002; 31: 817-22.

18. Menotti A, Puddu Pe, Lanti M. Comparison of the Framingham risk function-based coronary chart with risk function from an Italian population study. Eur Heart J 2000; 21: 365-70.

19. Marrugat J, Subirana I, Comín E, Cabezas C, Vila J, Elousa R ET AL. Validity of an adaptation of the Framingham cardiovascular risk function: the VERIFICA study. J Epidemiol Community Health 2007; 61: 40-7.

20. Hense HW, Schulte H, Löwel H, Assmann G, Keil U. Framingham risk function overestimates risk of coronary heart disease in men and women from Germany-results from the MONICA Augsburg and the PROCAM cohorts. Eur Heart J 2003; 24: 937-45.

21. World Health Organization. WHO Global Info Base World Health Statistics. Geneve, 2000. Versión online. Disponible en: http://www.who.int/infobase/ comparestart.aspx [Consultado el 20 marzo 2008].

22. Marrugat J, D'Agostino RB, Sullivan L, Elousa R, Wilson PW, Ordovas J et al. An adaptation of the Framingham coronary heart disease risk function to European Mediterranean areas. J Epidemiol Community Health 2003; 57: 634-8.

23. Departamento de Epidemiología. Ministerio de Salud. Encuesta Nacional de Salud 2003.

24. Organización Mundial de la Salud. Clasificación estadística internacional de enfermedades y problemas relacionados con la salud. - 10a. revisión. v. 3. Lista tabular. Washington, D.C.: OPS. (Publicación científica 554), 1995.

25. NúÑez L, Icaza G. Calidad de las estadísticas de mortalidad en Chile, 1997-2003. Rev Méd Chile 2006; 134: 1191-6.

26. Mathers C, Ma Fat D, Inoue M, Rao C, López A. Counting the dead and what they died from: an assessment of the global status of cause of death data. Bull World Health Organ 2005; 83: 171-177c.

27. Código Sanitario de la República de Chile, Santiago, Chile, Editorial Jurídica de Chile, Edición Oficial, Aprobado por Decreto $\mathrm{N}^{\mathrm{O}} 418$, de 18 de mayo de 2004, del Ministerio de Justicia. Disponible en: http://epi.minsal.cl/epi/html/RSI/VI/ 3 Miscelaneo/05codsan.pdf [Consultado el 26 de mayo 2008].
28. Marrugat J, Arboix A, García-Eroles L, Salas T, Vila J, Castell C et al. Estimación de la incidencia poblacional y la mortalidad de la enfermedad cerebrovascular establecida isquémica y hemorrágica en 2002. Rev Esp Cardiol 2007; 60: 573-80.

29. Álvarez A. Las tablas de riesgo cardiovascular. Una revisión crítica. MEDIFAM 2001; 11: 122-39.

30. Mendis S. Cardiovascular risk assessment and management in developing countries. Vasc Health Risk Manag 2005; 1: 15-8.

31. Population Division of the Department of Economic and Social Affairs of the United Nations Secretariat. World Population Prospects: The 2006 Revision and World Urbanization Prospects: The 2005 Revision. Disponible en: http://esa.un.org/unpp [Consultado el 20 mayo 2008].

32. D'Agostino RB, Vasan R, Pencina M, Wolf P, Cobain M, Massaro J et al. General cardiovascular risk profile for use in primary care. The Framingham Heart Study. Circulation 2008; 117: 743-53.

33. Haffner SM, Lehto S, Rönnemaa T, Pyörälä K, Laakso M. Mortality from coronary heart disease in subjects with type 2 diabetes and in nondiabetic subjects with and without prior myocardial infarction. N Engl J Med 1998; 339: 229-34.

34. Juutilainen A, Lehto S, Rönnemaa T, Pyörälä K, LaAkso M. Type 2 diabetes as a "coronary heart disease equivalent": an 18-year prospective population-based study in Finnish subjects. Diabetes Care 2005; 28: 2901-7.

35. Evans JMM, Wang J, Morris AD. Comparison of cardiovascular risk between patients with type 2 diabetes and those who had had a myocardial infarction: cross sectional and cohort studies. BMJ 2002; 324: 939-42.

36. Lee CD, Folsom AR, Pankow JS, Brancati FL. Atherosclerosis Risk in Communities (ARIC) Study Investigators. Cardiovascular events in diabetic and nondiabetic adults with or without history of myocardial infarction. Circulation 2004; 109: 855-60.

37. Hu FB, Stampfer MJ, Solomon CG, Liu S, Willett WC, Speizer FE ET AL. The impact of diabetes mellitus on mortality from all causes and coronary heart disease in women: 20 years of follow-up. Arch Intern Med 2001; 161: 1717-23.

38. Lotufo PA, Gaziano JM, Chae CU, Ajani UA, MorenoJohn G, Buring JE et al. Diabetes and all-cause and coronary heart disease mortality among US male physicians. Arch Intern Med 2001; 161: 242-7.

39. Cho E, Rimm EB, Stampfer MJ, Willett WC, Hu FB. The impact of diabetes mellitus and prior myocardial infarction on mortality from all causes and from coronary heart disease in men. J Am Coll Cardiol 2002; 40: 954-60.

40. Schramm TM, Gislason GH, Køber L, Rasmussen S, Rasmussen JN, Aвildstrøm SZ et al. Diabetes pa- 
tients requiring glucose-lowering therapy and nondiabetics with a prior myocardial infarction carry the cardiovascular risk. A population study of 3.3 million people. Circulation 2008; 117 : 1945-54.

41. González-Clemente J, Palma S, Arroyo J, Vilardell C, Caixàs A, Giménez-Palop O et al. ¿La diabetes mellitus es un equivalente de riesgo coronario? Resultados de un metaanálisis de estudios prospectivos. Rev Esp Cardiol 2007; 60: 1167-76.

42. Gerszten RE, WANG TJ. The search for new cardiovascular biomarkers. Nature 2008; 451: 949-52.

43. Direcció General de Salut Pública. Llibre blanc: Consens sobre les activitats preventives a l'edat adulta dins l'atenció primària, $2^{\mathrm{a}}$ ed. Barcelona: Generalitat de Catalunya, Departament de Salut; 2006. Disponible en: http://www.regicor.org/ fitxers_generals/actipreve2005.pdf [Consultado el 19 mayo 2008].
44. Grupo de Trabajo de Riesgo Cardiovascular de la Gerencia D’ÁAtenció PRimària de Mallorca. Riesgo cardiovascular: Guía actuación en Atención Primaria. Geréncia Atenció Primaria de Mallorca. Ib-salut. Baleares, 2007. Disponible en: http://www.regicor.org/publicacions/ Guia\%20RCV\%20Baleares.pdf [Consultado el 19 mayo 2008].

45. Senado de España. Boletín Oficial de las Cortes Generales Senado, VIII Legislatura, Serie I: Boletín General, 27 de abril de 2007, Número 699. Disponible en: http://www.senado.es/legis8/publicaciones/ html/maestro/index_I0699.html [Consultado el 28 abril 2008].

46. Protocolo del Estudio Verifica (Validez de la Ecuación de Riesgo Individual de Framingham de Incidentes Coronarios Adaptada) [manual en Internet]. Barcelona: 2002. Disponible en: http//www.regicor.org/ fitxers_generals/Protocolo\%20VERIFICA.pdf [Consultado el 20 mayo 2008]. 